\title{
Filopodial Adhesion Does Not Predict Growth Cone Steering Events In Vivo
}

\author{
Carolyn M. Isbister ${ }^{1,3}$ and Timothy P. O'Connor ${ }^{1,2,3}$ \\ Departments of ${ }^{1}$ Anatomy and ${ }^{2} Z o o l o g y ~ a n d ~{ }^{3}$ Graduate Program in Neuroscience, University of British Columbia, \\ Vancouver, British Columbia V6T 1Z3, Canada
}

\begin{abstract}
Migration of growth cones is in part mediated by adhesive interactions between filopodia and the extracellular environment, transmitting forces and signals necessary for pathfinding. To elucidate the role of substrate adhesivity in growth cone pathfinding, we developed an in vivo assay for measuring filopodial-substrate adhesivity using the well-characterized $\mathrm{Ti}$ pioneer neuron pathway of the embryonic grasshopper limb. Using time-lapse imaging and a combination of rhodaminephalloidin injections and Dil labeling, we demonstrate that the filopodial retraction rate after treatment with cytochalasin D or elastase reflects the degree of filopodial-substrate adhesivity. Measurements of filopodial retraction rates along regions of known differing substrate adhesivities confirmed the use of this assay to examine filopodial-substrate adhesion during in vivo pathfinding events. We analyzed 359 filopodia from $22 \mathrm{Ti}$ growth cones and found that there is no difference between the retraction rates of filopodia extending toward the correct target (on-axis) and filopodia extending away from the correct target
\end{abstract}

Growth cones of developing neurons must discriminate between a variety of environmental cues to accurately pathfind and establish connections with their targets. Filopodia extend and retract from the growth cone, actively exploring the environment and altering the direction of growth cone advance in response to these cues (for review, see Heidemann et al., 1990; Lin et al., 1994; Kater and Rehder, 1995; Lauffenburger and Horwitz, 1996; Mitchison and Cramer, 1996). Although it is established that filopodia are necessary for accurate growth cone guidance (Bentley and Toroian-Raymond, 1986; Chien et al., 1993; Zheng et al., 1996), it remains unclear how filopodia integrate and transduce guidance information from multiple external cues into motile forces.

Early in vitro experiments conducted by Letourneau (1975) indicated that growth cones may select the most adhesive pathway available for migration. These experiments supported a model for growth cone steering based on differential expression of adhesive molecules in the environment. More recently, various in vitro studies have quantified the adhesion of neurites on extracts of

\footnotetext{
Received July 16, 1998; revised Jan. 21, 1999; accepted Jan. 22, 1999.

This work was supported by the Medical Research Council Grant MT 13246 and the Natural Science and Engineering Council Grant OGP0171387. C.M.I. was supported by a University of British Columbia Graduate Fellowship. We are grateful to Dr. Paul J. Mackenzie for assistance in the statistical analysis and for critical reading of this manuscript.

Correspondence should be addressed to Dr. Carolyn M. Isbister, Department of Anatomy, 2177 Wesbrook Mall, University of British Columbia, Vancouver, British Columbia V6T 1Z3, Canada.

Copyright (C) 1999 Society for Neuroscience $\quad 0270-6474 / 99 / 192589-12 \$ 05.00 / 0$
}

(off-axis). These results indicate on-axis and off-axis filopodia have similar substrate adherence. Interestingly, we observed a $300 \%$ increase in the extension rates of on-axis filopodia during Ti growth cone turning events. Therefore, in addition to providing filopodia with important guidance information, regional cues are capable of modulating the filopodial extension rate. The homogeneity in filopodial retraction rates, even among these turning growth cones in which differential adhesivity might be expected to be greatest, strongly establishes that differential adhesion does not govern $\mathrm{Ti}$ pioneer neuron migration rate or pathfinding. We propose that the presence of local differences in receptor-mediated second messenger cascades and the resulting assembly of force-generating machinery may underlie the ability of filopodial contacts to regulate growth cone steering in vivo.

Key words: neuronal development; growth cones; filopodia; guidance mechanisms; motility; adhesion purified embryonic substrate-bound adhesion molecules (Gundersen, 1987, 1988; Calof and Lander, 1991; Lamoureux et al., 1992; Lemmon et al., 1992; Gomez and Letourneau, 1994). The majority of these studies showed little correlation between adhesion and neuron outgrowth, indicating that endogenous substrates may not exert their effects on axon guidance principally via relative adhesiveness. Nonetheless, observation of isolated growth cones on artificially simple substrates may not reflect in vivo mechanisms of growth cone guidance. However, in vivo growth cone adhesiveness has been difficult to quantify, and therefore, the role of adhesion in axonal pathfinding in vivo could only be inferred from observation of growth cone morphology and dynamics (Caudy and Bentley, 1986a; Myers and Bastiani, 1993) or in preparations devoid of extracellular matrix (Condic and Bentley, 1989a,b). Therefore, although it is accepted that adhesion to the substrate is necessary for normal outgrowth, the role of filopodial-substrate adhesion in directing axonal pathfinding in vivo remains unclear.

Axonal pathfinding has been studied extensively in the embryonic grasshopper limb where the Ti pioneer growth cones display a strong preference for migration along a stereotyped pathway (for review, see Bentley and O'Connor, 1992; Sanchez et al., 1995). Ti pioneer growth cone filopodia, which are necessary for accurate pathfinding, contact a variety of substrates including epithelial and neuronal cells and an extensive basal lamina (Bentley and Toroian-Raymond, 1986; Anderson and Tucker, 1988; Condic and Bentley, 1989a,b). In the present study we use timelapse imaging to demonstrate that regional cues along the $\mathrm{Ti}$ 
pioneer pathway not only provide guidance information but are capable of increasing extension rates of target-directed filopodia. To elucidate the role of substrate adhesion in these growth cone steering events, we developed an assay for quantifying substrate adhesivity for individual filopodia within a given Ti growth cone. Using this assay we examined whether there is a correlation between the direction of $\mathrm{Ti}$ pioneer growth cone extension and the filopodial-substrate adhesiveness. We demonstrate that in vivo growth cone steering events are not correlated with filopodial-substrate adhesion, thus indicating that differential filopodial adhesion to the extracellular environment does not govern growth cone pathfinding in vivo.

\section{MATERIALS AND METHODS}

Embryo culture and neuronal labeling. Schistocerca gregaria embryos were obtained from a colony maintained at the University of British Columbia. Eggs were staged and sterilized, and the Ti limb buds from 30.5 to $33.5 \%$ embryos were dissected as described previously (Bentley et al., 1979; Caudy and Bentley, 1986b). Embryos were placed ventral side down on a poly-L-lysine-coated coverslip $(6 \mathrm{mg} / \mathrm{ml})$; the dorsal epithelium of the Ti limb buds was cut lengthwise and unrolled flat to expose the pioneer pathway. A suction pipette was used to remove the mesodermal cells overlaying the limb epithelium, exposing the Ti neurons. Limb preparations were bathed in a modified RPMI culture medium and viewed with Nomarski optics on a Nikon inverted compound microscope (O'Connor et al., 1990). Ti neurons were labeled with DiI (Molecular Probes, Eugene, OR) by gently touching to the cell body a micropipette coated previously with DiI and air dried. Rhodamine-phalloidin (Molecular Probes) injections were performed as described previously (O'Connor and Bentley, 1993), and injected neurons were double labeled with $\mathrm{DiO}$ (Molecular Probes).

Time-lapse microscopy. Labeled Ti neurons were illuminated with a Nikon $100 \mathrm{~W}$ halogen light and the appropriate filter set (Chroma Tech) and were shuttered with a computer-controlled Lambda 10-2 shutter (Sutter Instruments). Neurons were imaged with a Princeton Instruments MicroMax CCD camera (Kodak chip KAF 1400) and digitized with Princeton Instruments Winview 1.6.2, with one exception (see Fig. 3 ) that was imaged with a Photometrics camera (Kodak chip KAF 1400) with Perceptics software. Elapsed time was automatically recorded for each image.

Three to five labeled Ti pioneer neurons were imaged until one growth cone was selected for full analysis. Growth cones displaying a complex array of filopodia required two to three focal planes imaged per time point, and the parts in focus were combined into a digital montage. To record in vivo growth cone behavior, we imaged labeled Ti neurons for 1-3 hr before cytochalasin D (CD) or elastase application. This time frame ensured that only healthy, normally advancing growth cones were selected for full analysis. A selected neuron was then imaged exclusively for 1-3 hr after the addition of drug. Healthy labeled Ti neurons in the dish not selected for full analysis were also imaged at the last time point before drug application, the first time point after drug application, and the final time point of the experiment. After image collection, preparations were fixed, and the Ti neurons and guidepost cells were labeled with neuron-specific antibodies to confirm their positions (Jan and Jan, 1982).

Cytochalasin D and elastase treatments. Cytochalasin D $(2 \mu \mathrm{M})$ or elastase $(0.03 \%)$ was introduced to limb preparations by replacing the original media in the dish with culture media containing the drug or enzyme (MacLean-Fletcher and Pollard, 1980; Bentley and ToroianRaymond, 1986; Forscher and Smith, 1988; Condic and Bentley, 1989a,b), and image collection then continued for 1-3 hr after treatment. In a few cases, growth cones were imaged for up to $12 \mathrm{hr}$ after media exchange. The time lapse between the last image before treatment and the first image after treatment was usually within $5 \mathrm{~min}$ and in many cases was as quickly as $1 \mathrm{~min}$.

Determining on-axis and off-axis filopodia. Growth cones were imaged at various developmental stages of the Ti pioneer pathway and categorized as either migrating within the intrasegmental femur epithelium or migrating within the trochanter segmental epithelium (Fig. 1). These growth cones were categorized further as either nonturning growth cones or growth cones in the process of turning. There are two abrupt stereotyped turning events in the Ti pioneer pathway. The first is at the $\operatorname{Tr}$ cell, located within the trochanter segmental epithelium, where

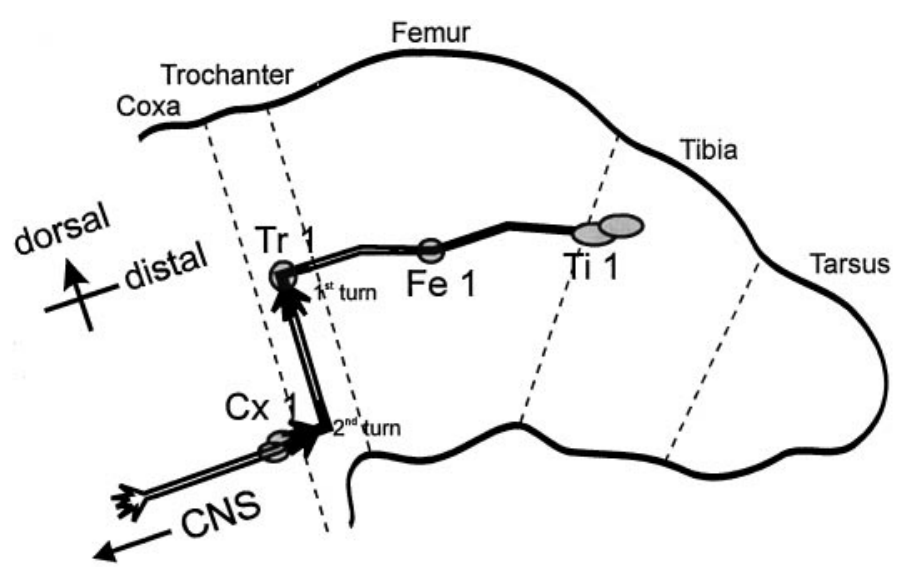

Figure 1. Schematic of the $T i$ pioneer neuron pathway. The pair of sibling $T i$ pioneer neurons arises from the underlying epithelium between 29 and $30 \%$ of embryonic development. At $\sim 30.5 \%$ of development, the $T i$ growth cones emerge from their cell bodies and begin to extend axons proximally along the limb axis toward the CNS. The Ti growth cones may or may not contact the $\mathrm{Fe} 1$ guidepost cell en route to the $\operatorname{Tr} 1$ cell. After contact with the $\operatorname{Tr} 1$ cell has been made, usually at $\sim 33 \%$ of development, the $T i$ growth cones reorient circumferentially (first turn) and extend ventrally along the trochanter epithelium. A second turning event in the $T i$ pioneer pathway occurs at $\sim 34 \%$ of development and is typified by a distinct reorientation toward the $C x 1$ guidepost cells. By $35 \%$, the $T i$ pioneer growth cones have extended proximally from the $C x 1$ cells into the CNS. Dashed lines indicate limb segment boundaries.

growth cones turn ventrally to migrate down the trochanter epithelium. The second is where growth cones turn proximally toward the Cx1 cells and migrate away from the trochanter segmental epithelium (Fig. 1).

Each filopodium was categorized as on- or off-axis. On-axis filopodia were those extending along the axis of the stereotyped Ti pioneer neuron migration trajectory (correct orientation); off-axis filopodia were those extending off the stereotyped migration axis by an angle of $>45^{\circ}$ (incorrect orientation; Fig. $2 A$ ). For growth cones in the process of turning ventrally at the $\operatorname{Tr} 1$ cell, on- and off-axis filopodia were often distinctly distributed into ventral and dorsal populations, respectively (Fig. 2B). Similarly, on- and off-axis filopodia of growth cones in the process of turning proximally toward the $\mathrm{Cx} 1$ cells were clearly distributed into proximal and distal populations, respectively (Fig. $2 C$ ). The location of the filopodial tip was the criterion for determining on- versus off-axis location; few filopodia had their base and tip in different categories (7 of 359).

Filopodial measurement and analysis. Filopodial length was measured for each time point using NIH Image. Growth cones that required multiple focal planes were digitally montaged to measure accurately the entire length of individual filopodia. Measurements were taken from the center of the growth cone, or branch, to the filopodial tip. Measuring from the center eliminated error from misinterpreting growth cone, or branch, diameter changes as filopodial length changes. Filopodia that retracted completely into the growth cone before addition of $\mathrm{CD}$ or elastase were not included. The results are based on the behavior of 359 filopodia from 22 growth cones for which we have complete data sets before and after media exchange.

Previous analysis of Ti filopodial behavior has determined that individual filopodia extend at relatively slow rates (on average $\sim 0.67 \mu \mathrm{m} /$ min) (O'Connor et al., 1990). This allowed us to sample filopodial lengths at relatively long time intervals $(10-15 \mathrm{~min})$, thus reducing the amount of exposure to the UV light source. However, to ensure that these time intervals were sufficient to reflect filopodial behavior accurately, we imaged some growth cones at higher frequencies. Also, because we could never be positive that filopodia did not extend and retract multiple times between acquired images, we calculated the net change in length over a period of $60 \mathrm{~min}$ before and after the addition of CD or elastase. Rates of extension and retraction over the $60 \mathrm{~min}$ before and after addition of drug were compared between on- and off-axis filopodial populations. For analysis of the difference in retraction rates between filopodial populations, we also compared length change during the first time period after drug application. A two-tailed unpaired $t$ test was used to compare the average retraction rate of all filopodia (both on- and 


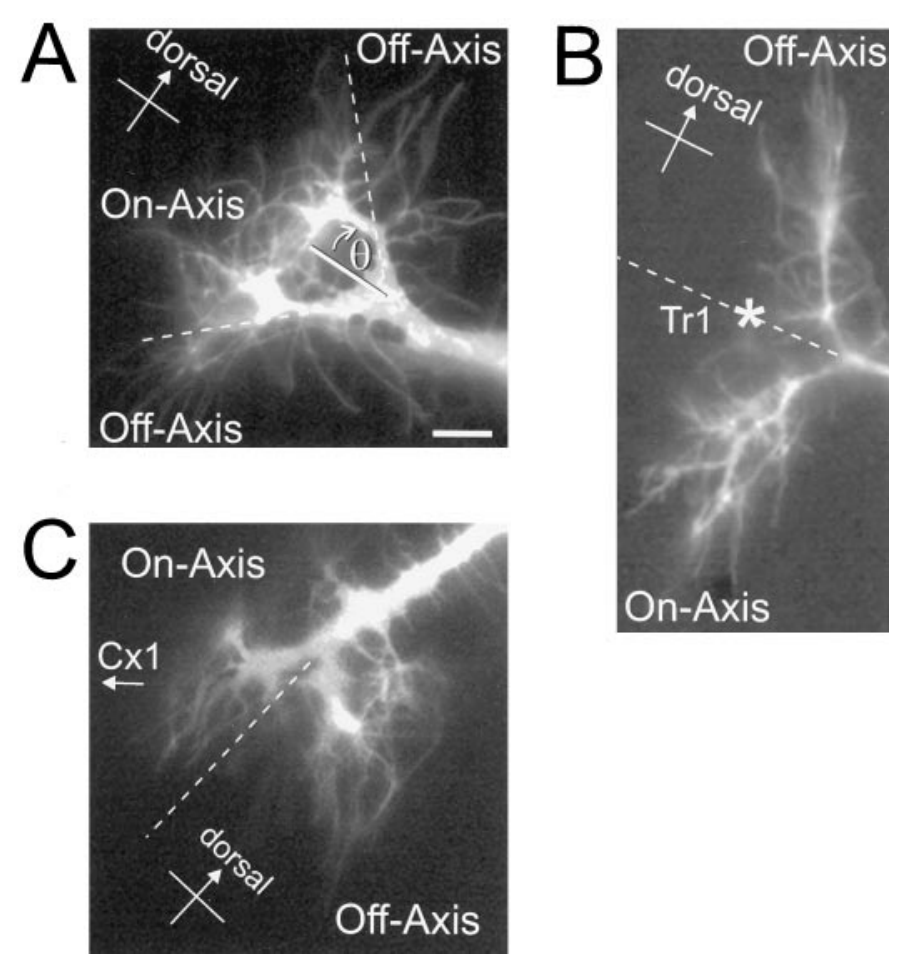

Figure 2. Categorization of growth cone filopodia as on- or off-axis. $A$, A representative nonturning $\mathrm{Ti}$ growth cone imaged during migration toward the $\operatorname{Tr} 1$ cell. On-axis filopodia are defined as those extending along the proximal and/or distal limb axis; off-axis filopodia are those extending off the stereotyped migration axis by an angle of $>45^{\circ}(\theta) . B$, A Ti growth cone imaged in the process of turning at the $\operatorname{Tr} 1$ cell $(*)$. Off-axis filopodia are those filopodia that are extended dorsally in the incorrect direction, whereas on-axis filopodia are defined as those filopodia that are extended in the correct ventral direction. This distribution of filopodia should maximize the chance of observing any adhesive differences between the ventral and dorsal epithelium. $C$, A Ti growth cone reorienting toward the $C x 1$ cells. As seen at the abrupt turn at the Tr cell, on- and off-axis filopodia are distinctly distributed into proximal and distal populations, respectively. Scale bar, $10 \mu \mathrm{m}$.

off-axis) from growth cones within the femur intrasegmental epithelium with the average retraction rate of all filopodia from growth cones within the trochanter segment boundary epithelium. For the remaining experiments, two-tailed unpaired $t$ tests were performed to compare on- and off-axis filopodia for individual growth cones. To pool data from all growth cones, we standardized the change in filopodial length (converted to $Z$ scores), and two-tailed unpaired $t$ tests were performed on the pooled standardized data.

\section{RESULTS}

\section{Cytochalasin D disrupts the internal cytoarchitecture of Ti filopodia}

Cytochalasins are fungal metabolites that inhibit filamentous actin (F-actin) elongation by capping actin filament ends in a reversible, dose-dependent manner (MacLean-Fletcher and Pollard, 1980; Schliwa, 1982; Cooper, 1987; Sampath and Pollard, 1991). Although inhibition of actin polymerization with cytochalasins blocks filopodia extension and motility (Marsh and Letourneau, 1984; Bentley and Toroian-Raymond, 1986), retrograde F-actin flow continues (Forscher and Smith, 1988). Previous in vitro studies have demonstrated that this continued F-actin retrograde flow, in the absence of polymerization, results in F-actin clearance from the growth cone lamellipodia and filopodia within 1-3 min, and usually after 2-4 min the filopodia have started to retract into the growth cone (Forscher and Smith, 1988).
Using rhodamine-phalloidin (Rh-phalloidin), we monitored actin dynamics in Ti filopodia after treatment with cytochalasin D. To confirm that CD disrupted F-actin in Ti growth cone filopodia, we injected selected $\mathrm{Ti}$ cell bodies with Rh-phalloidin, and the membrane was labeled with DiO. Images of F-actin dynamics and the growth cone morphology were collected before and after treatment with CD (Fig. 3). Immediately after CD addition, rhodamine-phalloidin-labeled actin began to disassemble and by 5-10 min was reduced to punctate staining within the filopodia. By $30 \mathrm{~min}$, Rh-phalloidin fluorescence was restricted to the growth cone body and large branches (Fig. 3D). Although CD rapidly removed the F-actin within the Ti filopodia, the majority of the filopodia remained extended (Fig. $3 C$ ). We attribute the slow retraction of $\mathrm{Ti}$ growth cone filopodia to the gradual loss of filopodial membrane adhesive contacts with the extracellular environment. In comparison with CD-treated growth cones in vitro, the Ti filopodia retract relatively slowly, possibly reflecting the complexity of the extracellular environment surrounding the filopodia. Filopodial retraction began 2-3 min after addition of $\mathrm{CD}$, thus suggesting that the mechanisms involved in generating filopodial retraction occur over the order of minutes. For many filopodia, retraction occurs over a number of hours (see Figs. 6, 7), with eventual complete retraction of the majority of filopodia (Fig. 4). For example, Figure 4 shows the distribution of filopodia extending from a growth cone that is migrating down the trochanter epithelium. After $12 \mathrm{hr}$ of exposure to CD, the growth cone had extended $\sim 15 \mu \mathrm{m}$; however only a few large filopodial branches remained. These branches were evenly dispersed around the growth cone with two on-axis and two off-axis branches remaining.

\section{Filopodial retraction rate after cytochalasin D or elastase application reflects filopodial-substrate adhesion}

Previous observations of growth cone morphology and direct tests of growth cone adhesion have illustrated that grasshopper trochanter segment epithelium is more adhesive than is femur intrasegmental epithelium (Caudy and Bentley, 1986a,b; Condic and Bentley, 1989a,b). To test the validity of our in vivo adhesion assay, we examined whether filopodial retraction rates after the addition of CD or elastase reflect these known differential adhesivities. We predicted that filopodia in contact with the more adhesive trochanter segment epithelium would retract slower after the removal of F-actin or the basal lamina adhesive interactions. To test this we compared the average filopodial retraction rate of growth cones migrating in the more adhesive trochanter epithelium with the average filopodial retraction rate of growth cones migrating in the less adhesive femur epithelium.

Figure 5 shows the average of both on- and off-axis filopodial retraction over the $60 \mathrm{~min}$ after $\mathrm{CD}$ or elastase application. For the CD trials, analysis of 60 filopodia from four growth cones migrating in the less adhesive femur epithelium revealed an average retraction of $10 \pm 0.6 \mu \mathrm{m}$ during the $60 \mathrm{~min}$ after $\mathrm{CD}$ application. In contrast, analysis of 87 filopodia from five growth cones migrating within the more adhesive trochanter epithelium exhibited a significantly slower average retraction of $7 \pm 0.8 \mu \mathrm{m}$ during the $60 \mathrm{~min}$ after $\mathrm{CD}$ application ( $t$ test, $p<0.001$ ). In addition, analysis of the averaged filopodial length change during the first time period after $C D$ addition revealed a significantly slower retraction rate of filopodia located in the trochanter epithelium ( $t$ test, $p<0.0005$; data not shown). Thus, slower filopodial retraction rates after $\mathrm{CD}$ application of growth cones migrat- 

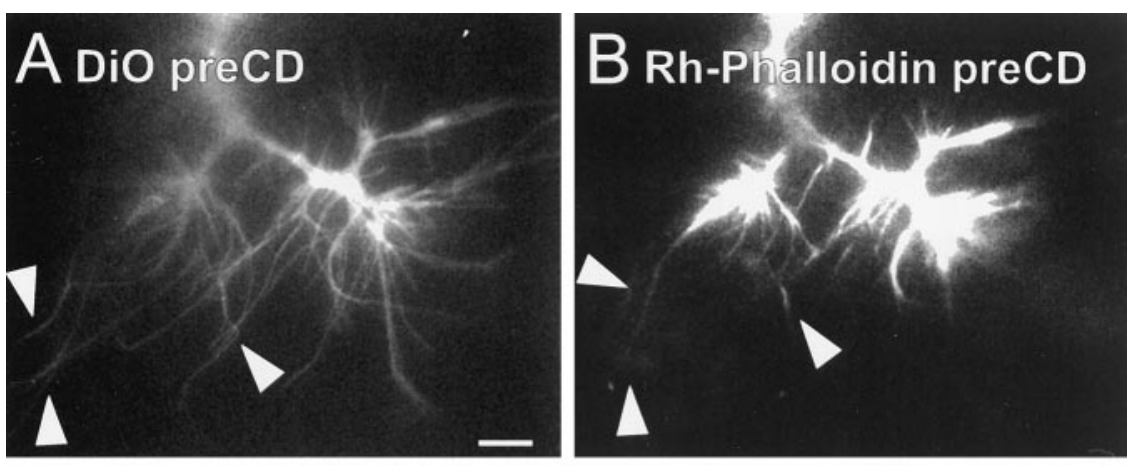

Figure 3. Cytochalasin D disrupts the internal cytoarchitecture of $\mathrm{Ti}$ filopodia. Images of a $\mathrm{Ti}$ neuron were collected as the growth cone migrated ventrally along the trochanter epithelium. Arrowheads demarcate the same three filopodia in all panels. $A, B$, Individual pioneer neurons were labeled with $\mathrm{DiO}$ to label the cell membrane $(A)$, and rhodaminephalloidin was intracellularly injected to label the F-actin $(B)$. Rh-phalloidin staining can be seen within the growth cone body and along the length of the filopodia ( $B$, arrowheads). $C, D i O$ image of the same growth cone displayed in $A 30$ min after $C D$ application is shown. Many of the filopodia remain extended ( $C$, arrowheads) despite the disruption of the actin cytoarchitecture with cytochalasin D. D, Rh-phalloidin image of the same growth cone $30 \mathrm{~min}$ after $C D$ treatment is shown. Rh-phalloidin staining was undetectable in filopodia ( $D$, arrowheads) and reduced to punctate staining within the growth cone body. Scale bar, $10 \mu \mathrm{m}$.
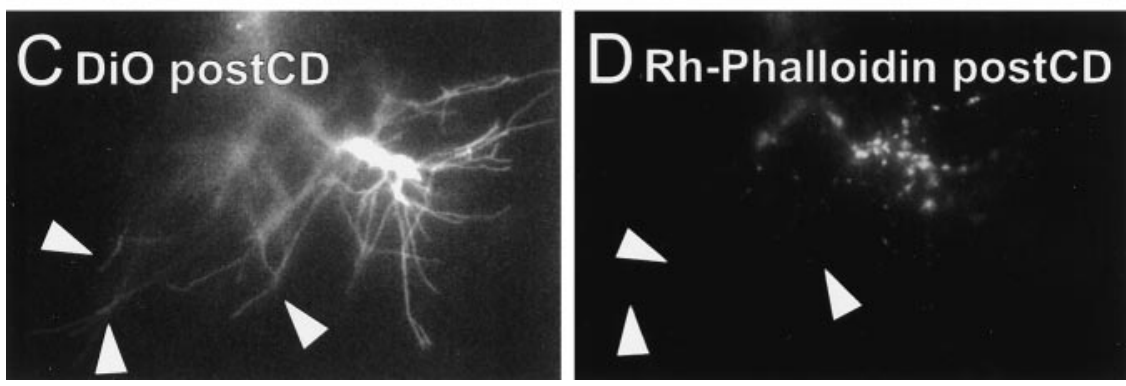

ing within trochanter segment epithelium accurately reflect filopodial contact with the more adhesive substrate.

Previous experiments have demonstrated that elastase disrupts growth cone-basal lamina adhesive contacts (Condic and Bentley, 1989a,b), thus leaving only the actin cytoskeleton and epithelial adhesive contacts to oppose filopodial collapse. Therefore, we confirmed the $\mathrm{CD}$ results by measuring Ti filopodial retraction rates after the removal of basal lamina adhesive interactions with elastase (Fig. 5). We analyzed four growth cones within the trochanter epithelium and three growth cones within the femur intrasegmental epithelium. We found, consistent with the CD results, that growth cones interacting with the more adhesive trochanter epithelium displayed slower filopodial retraction rates in comparison with the rates of filopodia extending along the less adhesive femur epithelium ( $t$ test, $p<10^{-5}$ ). These results support the use of the filopodial retraction rate as an assay of filopodial-substrate adhesivity during growth cone-steering events.

\section{Filopodial behavior of nonturning Ti pioneer neurons}

To record in vivo pathfinding behaviors of nonturning Ti pioneer neurons, we imaged growth cones for 1-3 hr after DiI labeling. Figure 6 is a representative example of the analysis for each nonturning growth cone in this study. In this example the growth cone was imaged as it migrated along the mid-femur epithelium toward the Tr1 cell (see Fig. 1). After the addition of CD, filopodia started to retract into the growth cone (Fig. 6 A, arrowheads). Analysis of individual filopodial length versus time illustrates that the majority of filopodia are dynamic, exhibiting periods of growth and retraction before CD treatment (Fig. 6B). On average, during the period of observation, length increased for both on- and off-axis filopodia in this particular growth cone, and no significant difference in the growth rate of on- and off-axis filopodia was observed $60 \mathrm{~min}$ before CD (Fig. $6 D$; $t$ test, $p=0.90$ ).

In the CD trials, we analyzed 11 nonturning growth cones $(n=$ 146 filopodia); in 9 of 11 growth cones, there was no significant difference between on- and off-axis filopodia extension during the 60 min before the addition of CD. In 1 of 11 growth cones, a significantly greater extension rate was observed in the on-axis filopodia during this period, whereas in 1 of 11 growth cones, a significantly greater extension rate was recorded for the off-axis filopodia. Thus, in the majority of nonturning growth cones, there was no significant difference in filopodial length changes observed between on- and off-axis filopodia before CD treatment. In addition, equal numbers of filopodia were distributed between onaxis and off-axis.

To pool the data from all nonturning growth cones, we standardized the change in length for each filopodia over the $60 \mathrm{~min}$ before $\mathrm{CD}$ application by conversion to $Z$ scores. Analysis of the pooled, standardized filopodia data confirmed there was no significant difference between on- and off-axis filopodial growth (see Fig. $8 A ; t$ test, $p=0.42$ ). The frequency histograms of the on- and off-axis pre-CD $Z$ scores were identically distributed, with filopodial length changes evenly distributed about their respective mean (data not shown). We separated the nonturning growth cones into subgroups based on the developmental stage of the $\mathrm{Ti}$ pathway, for example, before the Tr1 cell or along the trochanter epithelium. Analysis of these subgroups also revealed no significant difference in extension rates between on- and off-axis filopodia (before the Tr cell, $t$ test, $p=0.41$; trochanter epithelium, $t$ test, $p=0.83$ ). Likewise, analysis of the three nonturning growth cones from the elastase trials revealed no significant difference in filopodial extension rates (see Fig. 9C). These results suggest that in nonturning growth cones the dynamic behavior of on- and off-axis filopodia is not significantly different.

\section{Filopodial adhesion does not predict growth cone pathfinding of nonturning Ti neurons}

To determine the role of adhesion in Ti growth cone pathfinding in vivo, we compared the retraction rates of on- and off-axis filopodia after treatment with $\mathrm{CD}$ or elastase. We proposed that if adhesion of filopodia to the extracellular environment predicted growth cone migration, then on-axis filopodia should retract at a reduced rate when compared with off-axis filopodia. A representative example of the analysis of a nonturning growth cone from the CD trials is shown in Figure 6. All filopodia ceased extending, and a notable retraction was observed in both on- and off-axis filopodia (Fig. 6B,C). To determine whether there was a 

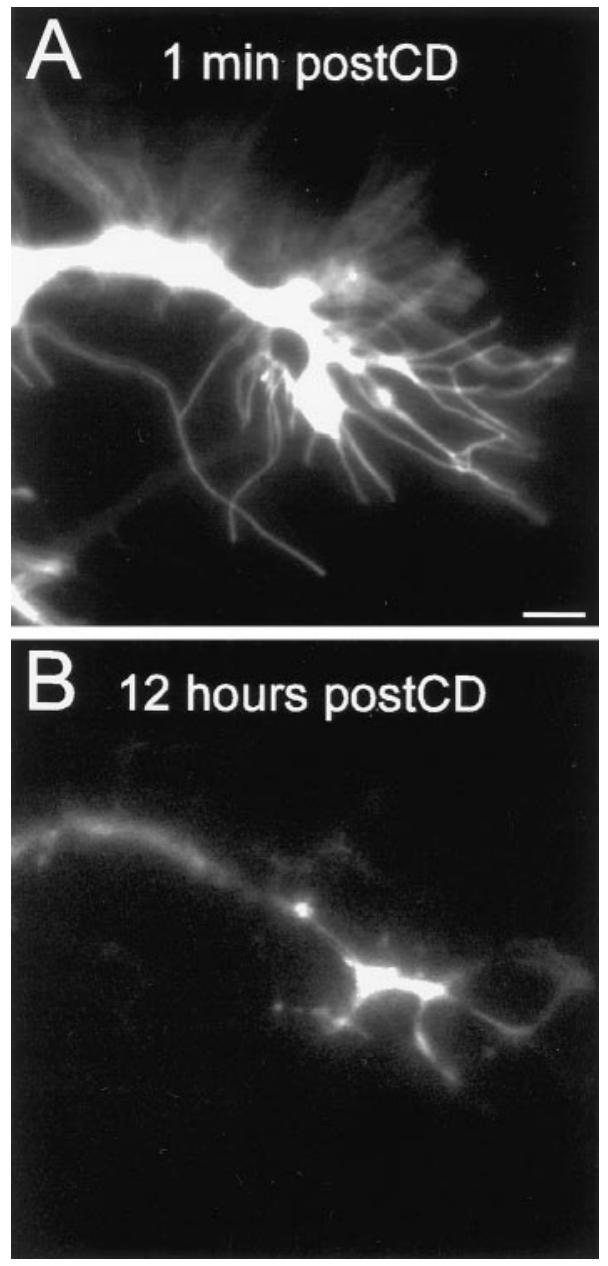

Figure 4. The majority of growth cone filopodia eventually retract after cytochalasin D treatment. Images of a DiI-labeled Ti neuron migrating along the trochanter epithelium are shown. $A$, One minute after $C D$ application, filopodia have not yet retracted into the growth cone. $B$, Twelve hours after $C D$ application, most of the filopodia have retracted into the growth cone. The growth cone has extended $\sim 15 \mu \mathrm{m}$. Scale bar, $10 \mu \mathrm{m}$.

significant difference between on- and off-axis retraction rates, we calculated the average change in filopodial length for the $60 \mathrm{~min}$ after CD application for both on- and off-axis filopodia. No significant difference was observed (Fig. $6 D$; $t$ test, $p=0.89$ ). In addition, analysis of the averaged filopodial length change during the first time period after $C D$ addition revealed no significant difference in retraction rates $(t$ test, $p=0.15)$.

For the CD trials, a total of 146 filopodia from 11 nonturning growth cones were analyzed before and after addition of CD. In 11 of 11 nonturning Ti neurons, analysis revealed no significant difference in retraction rates between on- and off-axis filopodia. Analysis of pooled, standardized filopodia also revealed no significant difference in filopodial retraction rates (see Fig. $8 B ; t$ test, $p=0.65)$. Furthermore, there was no significant difference in retraction rates even during the first time period after CD $(t$ test, $p=0.40)$ or when nonturning growth cones were grouped into developmental stages (before the $\operatorname{Tr}$ cell, $t$ test, $p=0.97$; ventral migration in trochanter epithelium, $t$ test, $p=0.67$ ). Representation of the post-CD $Z$ scores of nonturning growth cones as a frequency histogram revealed a shift in all on- and off-axis filopodial length changes to negative $Z$ scores (data not shown), con-

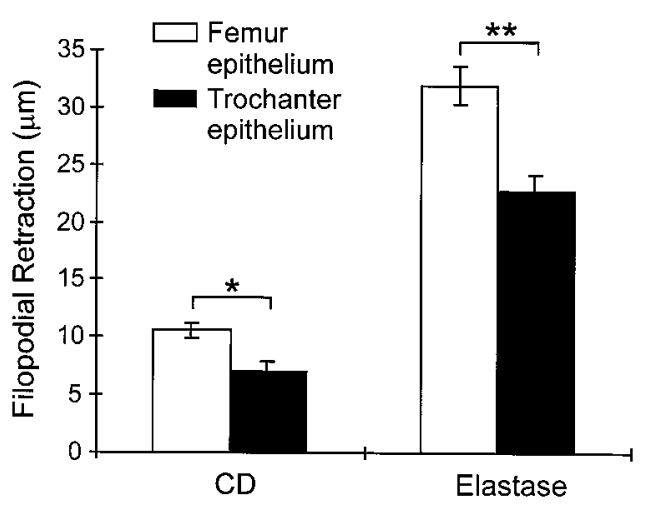

Figure 5. The filopodial retraction rate after cytochalasin D or elastase application accurately reflects in vivo substrate adhesion. Average filopodial retraction during $60 \mathrm{~min}$ after $C D$ application was determined for four growth cones migrating along the low-affinity (less adhesive) femur epithelium ( $n=60$ filopodia) and for five growth cones within the high-affinity (more adhesive) trochanter epithelium ( $n=87$ filopodia). Similarly, average filopodial retraction during $60 \mathrm{~min}$ after elastase application was determined for three growth cones migrating along the femur epithelium ( $n=66$ filopodia) and for four growth cones within the trochanter epithelium ( $n=69$ filopodia). The average retraction rate was determined using both on- and off-axis filopodia. After either $C D$ or elastase application, the average filopodial retraction rate for growth cones migrating along the less adhesive femur epithelium was significantly greater than the retraction rate for filopodia of growth cones interacting with the more adhesive trochanter segment epithelium $\left({ }^{*} p<0.001 ;{ }^{* *} p<\right.$ $10^{-5}$ ). Error bars indicate SEM.

firming that all filopodia began to retract after CD treatment. Analysis of the three elastase-treated nonturning growth cones also revealed no difference between the retraction rates of onand off-axis filopodia (see Fig. 9C). These data suggest that differential filopodial adhesion to the extracellular environment is not a governing factor in nonturning Ti neuron pathfinding in vivo.

\section{Filopodial behavior of Ti pioneer neurons during growth cone turning events}

The growth cones of $\mathrm{Ti}$ neurons that were in the process of turning were imaged for 1-3 hr before and after CD or elastase application. The data collection and analysis were identical to that of nonturning Ti neurons described above. Figure 7 is a representative example of the analysis of a turning growth cone from the $\mathrm{CD}$ trials. In this example a Ti growth cone turned proximally toward the $\mathrm{Cx} 1$ cells (Fig. $7 A$ ). As the main on-axis branch extending toward the $\mathrm{Cx} 1$ cells enlarged (Fig. $7 A$, open arrow), the large off-axis branch progressively collapsed (Fig. $7 \mathrm{~A}$, solid arrow). This shifting of the growth cone mass toward the main on-axis branch was accompanied by the extension of on-axis filopodia (Fig. $7 A$, arrowheads). Immediately after addition of $\mathrm{CD}$, what remained of the large off-axis branch continued to collapse (Fig. $7 A$, solid arrow), and the off- and on-axis filopodia began to retract (Fig. $7 A$, right, arrowheads). However, the main on-axis branch remained enlarged after CD application (Fig. $7 A$, right, open arrow). The persistence of the main on-axis branch after disruption of the actin cytoarchitecture most likely indicates that microtubules have invaded the main on-axis branch to form the emerging nascent axon (Sabry et al., 1991).

As observed with nonturning growth cones, filopodial number was evenly distributed on- and off-axis, and the majority of 

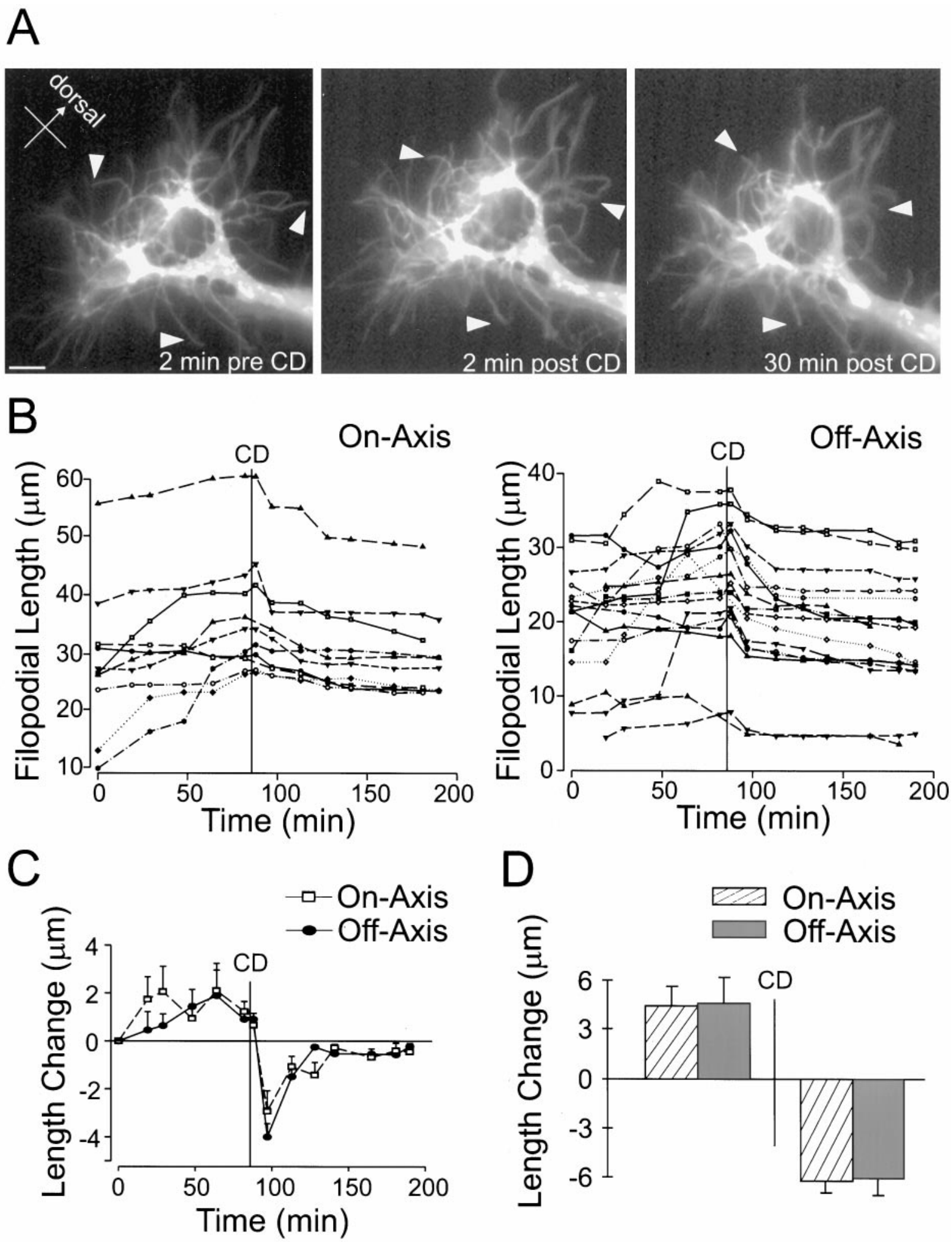

Figure 6. Pathfinding behavior of nonturning Ti growth cones before and after treatment with cytochalasin D. $A$, Three representative images of a DiI-labeled nonturning Ti growth cone migrating toward the Tr1 cell along the proximal-distal axis of the limb. Left, Before addition of CD. Middle, Two minutes after $C D$ treatment. Right, Thirty minutes after $C D$ treatment. Arrowheads demarcate the same filopodia before and after addition of $C D$ illustrating filopodial reaction. $B$, Filopodial length ( $y$-axis) versus time ( $x$-axis) for individual filopodia. Each trace indicates an individual filopodia. The time of $C D$ treatment is indicated by a vertical line. Left, On-axis filopodia. Right, Off-axis filopodia. $C$, Average change in filopodial length from the last time point ( $y$-axis) versus time ( $x$-axis). $C D$ treatment is indicated by a vertical line. Both on- and off-axis filopodia show immediate retraction after $C D$ application. $D$, Average change in filopodial length for $60 \mathrm{~min}$ before $(l e f t)$ and $60 \mathrm{~min}$ after $(r i g h t) C D$. No significant differences between on- and off-axis rates were observed before or after $C D$ treatment. Error bars indicate SEM. Scale bar, $10 \mu \mathrm{m}$. 
filopodia exhibited periods of growth and retraction before CD application (Fig. 7B). Surprisingly, when changes in filopodial length were calculated for each time interval and averaged for both on- and off-axis filopodia, a striking disparity in filopodial extension rates was observed between on- and off-axis filopodia during the $\mathrm{Ti}$ growth cone turning event. On-axis filopodia showed greater extension during the $60 \mathrm{~min}$ before $\mathrm{CD}$ application than did off-axis filopodia (Fig. $7 C, D ; t$ test, $p=0.03$ ). This disparity occurred in all of the turning growth cones examined. Analysis of the pooled $Z$ scores from a total of 78 filopodia from four growth cones revealed that this difference in on- versus off-axis extension during the $60 \mathrm{~min}$ before $\mathrm{CD}$ was consistent and highly significant only at turning points in the $\mathrm{Ti}$ pioneer pathway (Fig. $8 A$; $t$ test, $p<10^{-7}$ ). The frequency distribution of the pooled turning $Z$ scores revealed that both on- and off-axis $Z$ scores were evenly distributed about their respective means, with the on-axis filopodial mean 1.3 SDs greater than the off-axis filopodial mean (data not shown). This separation in the normally distributed on- and off-axis $Z$ scores indicated that the significant difference between extension rates was not the result of outliers (also confirmed by the height of the error bars in Fig. 8).

We analyzed the pooled raw data to determine whether the statistical difference observed between on- and off-axis filopodial extension before $\mathrm{CD}$ in turning growth cones was a result of increased growth in the on-axis filopodia or a relative decrease in off-axis filopodial extension. This analysis revealed that the average on-axis filopodial extension rate was $>300 \%$ greater than the average off-axis filopodial extension rate (Fig. $8 B$; $t$ test, $p<$ $\left.10^{-5}\right)$. Interestingly, this increase in on-axis extension in turning growth cones was also significantly greater $(>100 \%)$ than that in on-axis filopodial extension in nonturning growth cones (Fig. $8 B$; $t$ test, $p=0.005)$. There was no significant difference in the off-axis filopodial extension rates of turning and nonturning growth cones (Fig. $8 B ; t$ test, $p=0.11$ ). Furthermore, analysis of the four turning growth cones in the elastase trials also revealed these trends (Fig. 9; $n=69$ filopodia), increasing the number of turning growth cones to eight. These results indicate that in vivo Ti pioneer neuron turning events are characterized by an increased on-axis filopodial extension rate during turning events.

\section{Filopodial adhesion does not predict Ti growth cone turning events}

Although Ti growth cones exhibit a number of different morphologies at decision points (O'Connor et al., 1990), we found that filopodia were evenly distributed between on- and off-axis sectors, thus suggesting that asymmetric distribution of filopodia does not predict growth cone steering events. Considering that filopodial-substrate adhesion is greater in the regions where $\mathrm{Ti}$ growth cones commit to turning decisions, for example, at the $\mathrm{Tr}$ cell in the trochanter epithelium, we hypothesized that a differential adhesion gradient across the growth cone may underlie the growth cone turning event and the observed increase in filopodial extension. Therefore, to determine the role of filopodial adhesivity in Ti growth cone turning, we compared the retraction rates of on- and off-axis filopodia after treatment with CD or elastase.

Figure 7 illustrates a representative example of the analysis of a turning growth cone from the CD trials. Consistent with nonturning growth cones, filopodia ceased extending and began retracting into the growth cone after $\mathrm{CD}$ application (Fig. $7 B, C$ ). To determine whether there was a significant difference between on- and off-axis retraction rates, we calculated the average change in filopodial length for the $60 \mathrm{~min}$ after $\mathrm{CD}$ application. No significant difference was observed (Fig. $7 D ; t$ test, $p=0.33$ ), thus suggesting that the greater net extension of on-axis filopodia was not caused by greater filopodial-substrate adhesiveness. In addition, analysis of the averaged filopodial length change during the first time period after $\mathrm{CD}$ addition confirmed there was no significant difference in filopodial retraction rates ( $t$ test, $p=0.14$ ).

We analyzed four turning growth cones in the CD trials $(n=$ 78 filopodia); in all $\mathrm{Ti}$ growth cones examined, there was no significant difference in retraction rates between on-axis filopodia and off-axis filopodia. Analysis of the pooled $Z$ scores confirmed there was no significant difference in retraction rates during the 60 min after CD application (Fig. $8 A ; t$ test, $p=0.40$ ) or during the first time period after $\mathrm{CD}(t$ test, $p=0.96)$. In addition, as seen in nonturning growth cones, the frequency histogram demonstrated a consistent shift in all filopodial length changes to negative $Z$ scores (data not shown). Furthermore, we analyzed four turning growth cones in the elastase trials ( $n=69$ filopodia); again consistent with the $\mathrm{CD}$ results, there was no significant difference between on- and off-axis retraction rates (Fig. 9). These results indicate that Ti growth cone steering events and the corresponding increase in on-axis filopodial migration rate during these turning events are not directed by differential filopodial-substrate adhesion.

\section{DISCUSSION}

Using the well-characterized Ti pioneer neuron pathway of the embryonic grasshopper limb, we developed an assay to test whether differences in filopodial adhesion direct growth cone steering events in vivo. We found that although filopodia in general exhibit robust adhesive interactions with the surrounding extracellular environment, there is no evidence that differential filopodial adhesion directs $\mathrm{Ti}$ growth cone steering events in vivo. In addition, we found that in vivo regional cues not only provide filopodia with essential guidance information but are also capable of modulating filopodial extension rate.

\section{Retraction rates of filopodia lacking F-actin or basal lamina interactions as a measure of filopodial-substrate adhesivity}

Traditionally, in vivo growth cone-substrate adhesion has been difficult to address because of the complexity of the in vivo environment and the impediments to access and manipulation. Thus, the role of substrate adhesion in growth cone pathfinding has remained unclear. In the present study, we took advantage of the accessibility of the well-characterized embryonic grasshopper limb Ti pioneer neuron projection to develop an assay for quantifying in vivo filopodial-substrate adhesivity. Our assay uses the filopodial retraction rate, after the disruption of either the actin cytoarchitecture with $\mathrm{CD}$ or the basal lamina adhesive interactions with elastase, as a measure of substrate adhesivity. To confirm the validity of our assay, we compared the filopodial retraction rates of growth cones migrating along substrates of known differing adhesivity. Evidence from observations of $\mathrm{Ti}$ growth cone morphology and from direct tests of growth cone adhesion suggests that epithelium in the trochanter limb segment is more adhesive than is intrasegmental femur epithelium (Caudy and Bentley, 1986a,b; Condic and Bentley, 1989a,b). Using our in vivo adhesion assay, we demonstrate that filopodia of Ti growth cones migrating within the more adhesive trochanter epithelium retract significantly slower than do filopodia of growth cones migrating within the less adhesive femur epithelium. Thus, our assay of filopodial retraction rate after application of $\mathrm{CD}$ or elastase accurately reflects in vivo filopodial-substrate adhesion. 
A
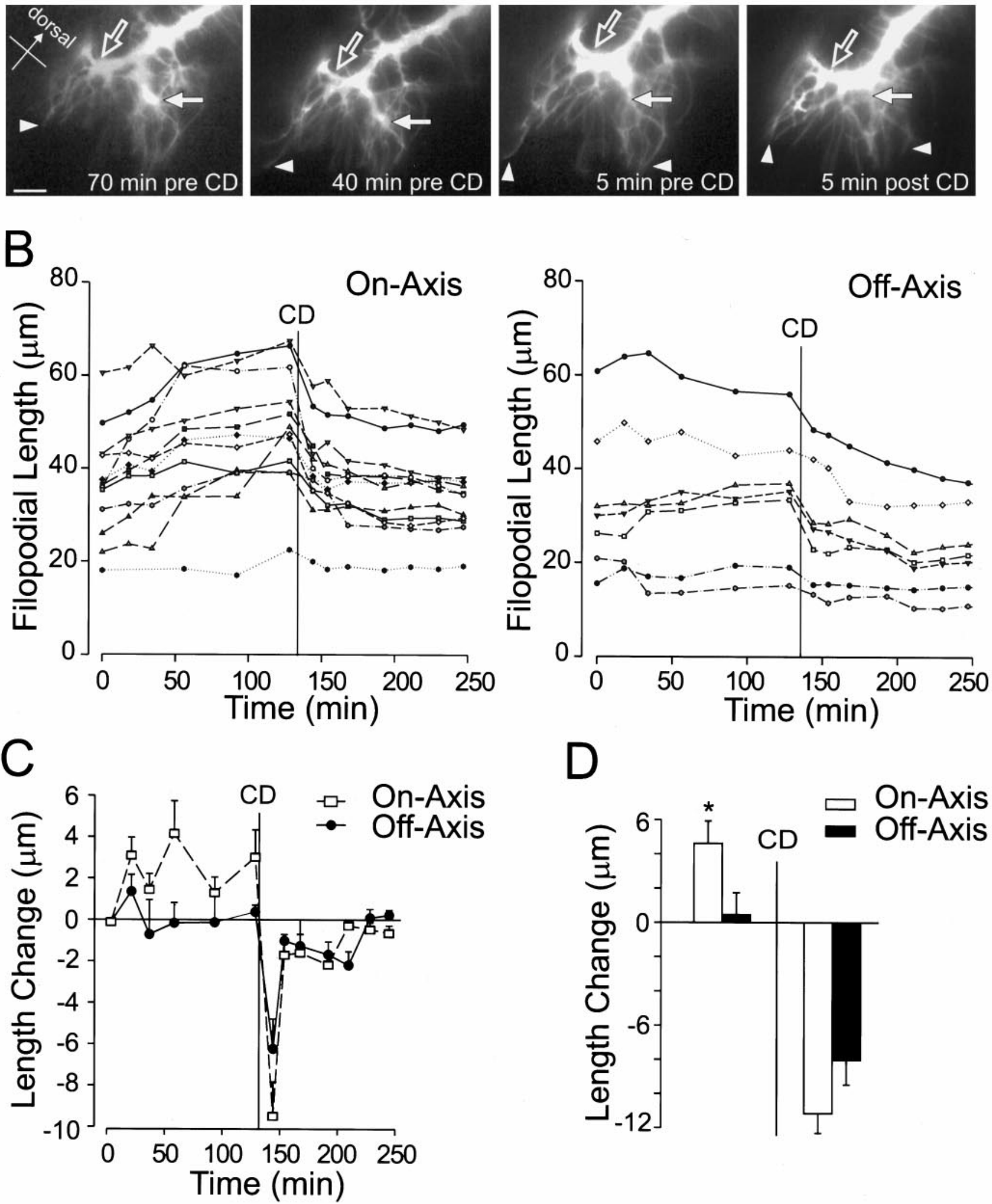

$\mathrm{D}$

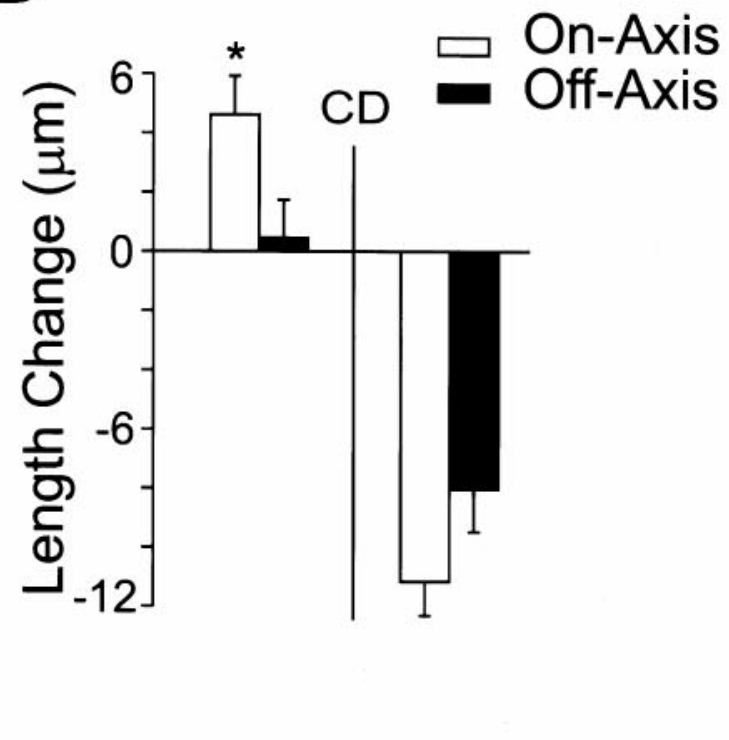

Figure 7. Pathfinding behavior of a Ti growth cone turning toward the Cx1 cells before and after treatment with cytochalasin D. $A$, Four representative images of a DiI-labeled Ti growth cone turning toward the Cx1 cells. Left, Middle, Before addition of cytochalasin D Right, Five minutes after $C D$ treatment. Arrowheads demarcate filopodial extension before and retraction after addition of cytochalasin D. The main on-axis (open arrow) and main off-axis (solid arrow) branches are indicated. After addition of cytochalasin D, the large off-axis branch has narrowed (Figure legend continues) 
A Pooled Z-Scores

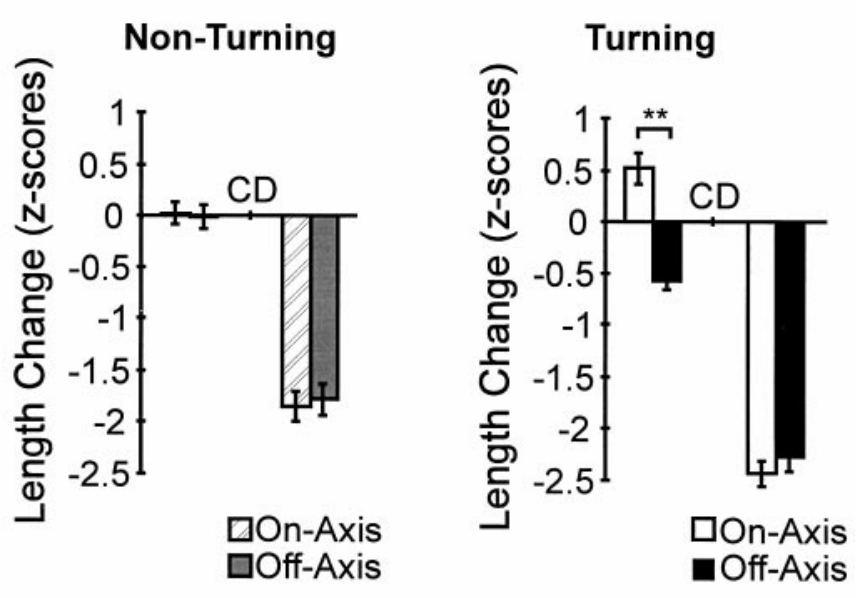

B Pooled Raw Data

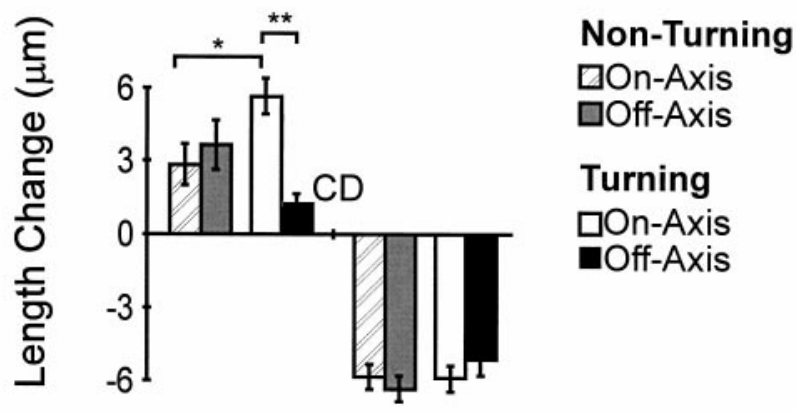

Figure 8. In vivo Ti growth cone pathfinding behaviors and filopodial extension rate are not governed by differential filopodial-substrate adhesion. $A$, The average standardized length change ( $Z$ score) $60 \mathrm{~min}$ before (left in each panel) and 60 min after (right in each panel) $C D$ application for 11 nonturning and 4 turning growth cones $(n=224$ filopodia). There was no significant difference between on- and off-axis filopodial growth in nonturning growth cones during the $60 \mathrm{~min}$ before $C D$ application. However, a highly significant difference between on- and off-axis growth $60 \mathrm{~min}$ before $C D$ application was observed in turning growth cones $(n=$ 78 filopodia; $\left.{ }^{* *} p<10^{-7}\right)$. There was no significant difference between onand off-axis filopodial length change observed in nonturning or turning growth cones after $C D$ application. $B$, Average length change for $60 \mathrm{~min}$ before (left) and $60 \mathrm{~min}$ after (right) $C D$ application calculated using nonstandardized raw data (the similarity in frequency distributions determined by Kolmogorov-Smirnov tests). The statistical difference observed between on- and off-axis filopodial extension in turning growth cones was a result of increased growth in the on-axis filopodia versus a relative decrease in off-axis filopodial extension $\left(* * p<10^{-5}\right)$. On-axis filopodial extension during growth cone turning events is significantly greater than is on-axis extension during nonturning events $\left({ }^{*} p<0.01\right)$. No significant difference was observed in the raw data between on- and off-axis filopodial retraction after addition of $C D$ for either nonturning or turning growth cones. Error bars indicate SEM.
Our assay is based on the premise that the combined action of the actin cytoskeleton and adhesion opposes tension within filopodia, preventing them from collapsing into the body of the growth cone. With the loss of the actin cytoskeleton after CD treatment, the adhesive contacts are not sufficient to oppose tension within filopodia, and therefore filopodia retract into the growth cone at a rate dependent on the degree of filopodial-substrate adhesivity. Likewise, after removal of the basal lamina adhesive interactions with elastase, the actin cytoskeleton and underlying epithelial adhesive contacts are insufficient to maintain filopodial extension.

Although the integrity of F-actin is important for force generation during filopodial extension, several lines of evidence indicate a network of F-actin is not necessary for Ti filopodialsubstrate adhesion. First, it has been well established in many systems, including the grasshopper Ti pioneer pathway, that an intact actin cytoarchitecture is not required for growth cone adhesion (Marsh and Letourneau, 1984; Bentley and ToroianRaymond, 1986; Letourneau et al., 1987; Forscher and Smith, 1988; Chien et al., 1993; Zheng et al., 1996). In fact, in the absence of actin, growth cones have been shown to remain adhered to a substrate and are even capable of extension (Marsh and Letourneau, 1984; Bentley and Toroian-Raymond, 1986; Chien et al., 1993; Zheng et al., 1996). In addition, filopodial and lamellipodial retraction often lags behind the loss of F-actin. For example, numerous Aplysia growth cone filopodia remain extended after the addition of cytochalasins, in some cases for up to 30 min [see Forscher and Smith (1988), their Fig. 7D]. Second, a variety of classical adhesive receptor complexes are known to exhibit adhesive interactions that are not associated with the actin cytoskeleton (Regen and Horwitz, 1992; Schmidt et al., 1993; Hortsch et al., 1995; Shapiro et al., 1995; Dubreuil et al., 1996; Kreft et al., 1997). Third, although electron microscopic examination of the actin distribution in the body of neuronal growth cones has identified interactions along the length of F-actin bundles with intramembranous particles (Lewis and Bridgman, 1992), similar interactions were not reported along the length of filopodia. Finally, although we cannot comment on the molecular nature of Ti filopodial-substrate adhesion, the maintenance of filopodial extension after CD clearly demonstrates continued filopodial adhesion to the extracellular environment in the absence of the actin cytoarchitecture.

\section{Differential adhesion as a model of neuronal pathfinding}

The Ti growth cone filopodia interact with a variety of substrates including the extracellular matrix, epithelial cells, and several preaxonogenesis neurons. These substrates express a variety of substrate-bound guidance molecules, some of which have been shown to be necessary for accurate $\mathrm{Ti}$ growth cone pathfinding (Bentley and Caudy, 1983; Caudy and Bentley, 1986a; Kolodkin et al., 1992; Sanchez et al., 1995; Tsai et al., 1997; Wong et al., 1997). How these substrate-bound adhesion molecules interact with Ti filopodia to direct growth cone steering events in vivo is not well understood. One mechanism that may direct growth cone motility is differential filopodial-substrate adhesion. Increased

\section{$\leftarrow$}

markedly (solid arrow), whereas the principal on-axis branch remains enlarged, possibly indicating microtubule invasion and nascent axon formation (open arrow). B, Filopodial length ( $y$-axis) versus time ( $x$-axis) for individual filopodia. The time of $C D$ treatment is indicated by a vertical line. Left, On-axis filopodia. Right, Off-axis filopodia. $C$, Average change from the last time point in filopodial length ( $y$-axis) versus time ( $x$-axis). The time of $C D$ application is indicated by the vertical line. $D$, Average change in filopodial length for 60 min before (left) and 60 min after (right) $C D$. A significant difference between on- and off-axis filopodial growth was observed before addition of $C D\left({ }^{*} p<0.05\right)$. No significant difference was observed between on- and off-axis filopodial retraction after addition of $C D$. Error bars indicate SEM. Scale bar, $10 \mu \mathrm{m}$. 
A
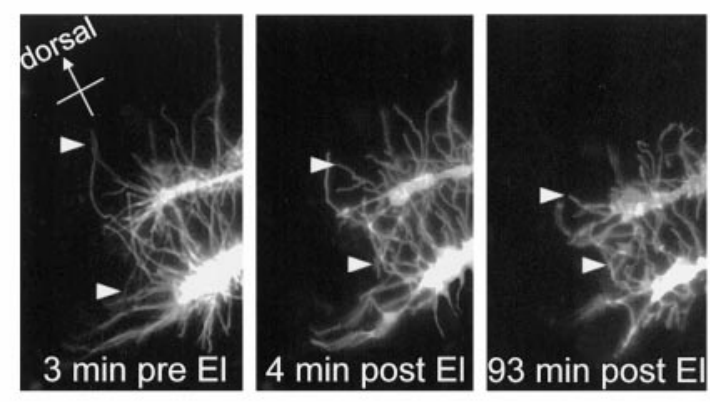

\section{Pooled Raw Data}

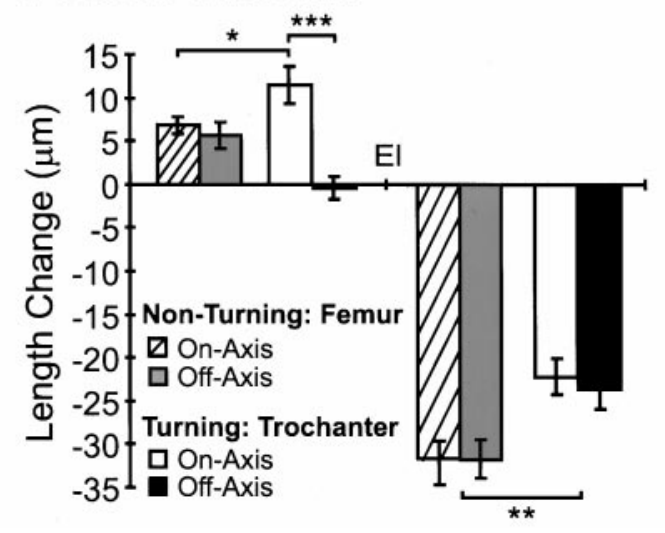

B

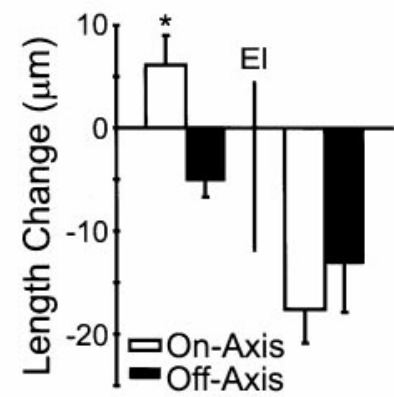

Figure 9. Elastase experiments confirm that the in vivo filopodial extension rate and pathfinding behaviors of $\mathrm{Ti}$ growth cones are not governed by differential filopodialsubstrate adhesion. $A$, Three representative images of a pair of sibling DiI-labeled Ti growth cones in the process of turning ventrally within the trochanter. The analysis presented in $B$ is of the top growth cone. Left, Before addition of elastase $(E l)$. Middle, Right, After elastase treatment. Arrowheads demarcate the same filopodia before and after addition of elastase illustrating filopodial retraction. $B$, Average change in filopodial length for $60 \mathrm{~min}$ before (left) and $60 \mathrm{~min}$ after (right) elastase. A significant difference between on- and off-axis filopodial growth was observed before addition of elastase $\left({ }^{*} p<0.005\right)$. No significant difference was observed between on- and off-axis filopodial retraction after addition of elastase. $C$, Pooled elastase data from seven growth cones (135 filopodia), illustrating the average length change for $60 \mathrm{~min}$ before (left) and $60 \mathrm{~min}$ after (right) elastase application (calculated using nonstandardized raw data; the similarity in frequency distributions determined by Kolmogorov-Smirnov tests). The on-axis filopodia of turning growth cones extend at an increased rate $\left({ }^{* * *} p<10^{-6} ; * p<0.05\right)$; however, no significant difference was observed between the on- and off-axis retraction rates after elastase for either turning or nonturning growth cones. In the elastase trials, all nonturning growth cones were migrating in the femur, and all turning growth cones were at the Tr cell within the trochanter segment epithelium. Therefore, the pooled raw data also reveal the significantly slower filopodial retraction of growth cones interacting with the more adhesive trochanter epithelium $\left(* * p<10^{-5}\right)$. Error bars indicate SEM.

filopodial-substrate adhesion may be a consequence of a greater number of substrate-bound molecules binding to filopodial receptors, leading to an increased receptor coupling to the actin cytoskeleton and a slowing of the retrograde F-actin flow. Growth cone advance, therefore, could result from attenuation of the retrograde $\mathrm{F}$-actin flow combined with continued actin polymerization at the leading edge (Lin and Forscher, 1995). Alternatively, an increased receptor coupling to the actin cytoskeleton could manifest itself intracellularly as increased tension within filopodia, creating more traction force to "pull" the growth cone forward (Heidemann et al., 1990). Regardless of the cytomechanics, a differential expression of adhesive molecules in the environment, their respective receptors on filopodia, or alterations in the ligand-receptor binding affinity could reorient the growth cone and alter pathfinding.

Because it was the focus of the present study to determine whether adhesion alone is sufficient to determine growth cone steering events in vivo, we used our in vivo adhesion assay to test whether an increased substrate adhesivity could predict Ti growth cone pathfinding behaviors. If differential adhesion across the growth cone governs Ti pathfinding in vivo, then correctly oriented (on-axis) and incorrectly oriented (off-axis) filopodial retraction rates should differ after $\mathrm{CD}$ or elastase treatment. We found that filopodial retraction rates did not differ between onand off-axis filopodia at any of the positions measured along the pathway, including during the committed turning events. The homogeneity in filopodial retraction rates, even among turning growth cones in which we would predict that differential adhesion should be greatest, strongly indicates that differential adhesion does not determine $\mathrm{Ti}$ pioneer neuron steering in vivo. The complexity and heterogeneity among in vivo guidance mechanisms, however, caution against prematurely concluding that differential substratum adhesivity has no role in every in vivo growth cone guidance situation. For example, although our results establish that spatial differences in filopodia-substrate adhesion are not sufficient to determine $\mathrm{Ti}$ pioneer growth cone pathfinding, there may exist in vivo situations in which spatial heterogeneity in substratum adhesion across the body of a growth cone is sufficient to regulate steering events.

In this study filopodial and growth cone behaviors were also examined for at least $1 \mathrm{hr}$ before the addition of CD or elastase. This provided us with information about in vivo growth cone pathfinding behaviors at various decision points along the $\mathrm{Ti}$ pioneer pathway. We observed no consistent disparity between on- and off-axis extension rates in the nonturning growth cones. To our surprise, however, we observed a threefold increase in on-axis filopodial extension rate during Ti growth cone-turning events. Furthermore, when the on-axis filopodial extension rate for turning growth cones was compared with the extension rate for on-axis filopodia of nonturning growth cones, the difference was still significant; the turning filopodia extended nearly twice the distance during the $60 \mathrm{~min}$ before drug. Interestingly, a previous in vitro study demonstrated that model guideposts, composed of laminin-coated beads, not only provide directional guidance information to dissociated dorsal root ganglion neurons but also cause a sustained 2.5-fold increase in growth cone velocity (Kuhn et al., 1995). This study and our in vivo pathfinding results indicate that at decision points local environmental cues may induce growth cones to change migration rate and direction. 


\section{Alternative models of growth cone guidance}

We have established that differential filopodial adhesion to the extracellular environment is insufficient to direct Ti growth cone pathfinding events. These results raise the question of what alternative cellular mechanisms could guidance molecules initiate to induce growth cone steering events in vivo. Evidence from in vitro studies suggests that the binding of filopodial receptors to extracellular matrix molecules may produce intracellular signaling cascades that modulate growth cone pathfinding. Many intracellular regulatory mechanisms, including kinases and phosphatases (Brambilla and Klein, 1995; Chang et al., 1995; Desai et al., 1996; Krueger et al., 1996; Gallo et al., 1997; He et al., 1997), calcium concentration (Williams et al., 1992; Gomez et al., 1995; Davenport et al., 1996; Kuhn et al., 1998), phospholipase C $\gamma$ (Saffell et al., 1997), cAMP (Kim and Wu, 1996; Song et al., 1997), and the small GTP-binding proteins (Kuhn et al., 1997; Luo et al., 1997; Hall, 1998), have been implicated in growth cone motility. In addition, it has been demonstrated that the concentration of substratum-bound ligand can post-translationally regulate the amount of receptor expressed on the surface of neurons (Condic and Letourneau, 1997). Therefore, although much is yet to be learned about the relationships between local second messenger cascades and subsequent changes in cytoskeletal organization and membrane adhesion, second messenger systems may regulate the assembly and interaction of force-generating machinery within pathfinding growth cones.

Our data demonstrate a uniform increase in filopodia-substrate adhesion at the trochanter segment epithelium; however the growth cone turning events and increases in filopodial extension observed at this decision point are not the result of differential filopodial adhesion across the growth cone. If the only role for substrate adhesivity is to ensure that migrating growth cones remain closely apposed to the substrate, why would there be an increase in substrate adhesivity at the trochanter? Previous observations have shown that $\mathrm{Ti}$ growth cones radically alter their morphology after migrating onto the trochanter epithelium (O'Connor et al., 1990). Growth cones typically extend branches and filopodia along the trochanter epithelium, effectively increasing their sampling area along the dorsal-ventral axis (O'Connor et al., 1990). It is possible that increases in substrate adhesivity at growth cone decision points, such as the trochanter segment epithelium, are responsible for these changes in growth cone morphology. An alteration in growth cone morphology could effectively increase the probability of filopodial contact with guidance cues in the environment. Therefore, one alternative role for substrate adhesivity may be to alter the morphology of growth cones to ensure filopodial interaction with guidance cues.

\section{REFERENCES}

Anderson H, Tucker RP (1988) Pioneer neurones use basal lamina as a substratum for outgrowth in the embryonic grasshopper limb. Development 104:601-608.

Bentley D, Caudy M (1983) Pioneer axons lose directed growth after selective killing of guidepost cells. Nature 304:62-65.

Bentley D, O'Connor TP (1992) Guidance and steering of peripheral pioneer growth cones in grasshopper embryos. In: The nerve growth cone (Letourneau PC, Kater SB, Macagno ER, eds), pp 265-282. New York: Raven.

Bentley D, Toroian-Raymond A (1986) Disoriented pathfinding by pioneer neurone growth cones deprived of filopodia by cytochalasin treatment. Nature 323:712-715.

Bentley D, Keshishian H, Shankland M, Toroian-Raymond A (1979)
Quantitative staging of embryonic development of the grasshopper Schistocerca nitens. J Embyol Exp Morphol 54:47-74.

Brambilla R, Klein R (1995) Telling axons where to grow: a role for EPH receptor tyrosine kinases in guidance. Mol Cell Neurosci 6:487-492.

Calof AL, Lander AD (1991) Relationship between neuronal migration and cell-substratum adhesion: laminin and merosin promote olfactory neuronal migration but are anti-adhesive. J Cell Biol 115:779-794.

Caudy M, Bentley D (1986a) Pioneer growth cone morphologies reveal proximal increases in substrate affinity within leg segments of grasshopper embryos. J Neurosci 6:364-379.

Caudy M, Bentley D (1986b) Pioneer growth cone steering along a series of neuronal and non-neuronal cues of different affinities. J Neurosci 6:1781-1795.

Chang HY, Takei K, Sydor AM, Born T, Rusnak F, Jay DG (1995) Asymmetric retraction of growth cone filopodia following focal inactivation of calcineurin. Nature 376:686-690.

Chien C-B, Rosenthal DE, Harris WA, Holt CE (1993) Navigational errors made by growth cones without filopodia in the embryonic $\mathrm{Xe}$ nopus brain. Neuron 11:237-251.

Condic ML, Bentley D (1989a) Removal of the basal lamina in vivo reveals growth cone-basal lamina adhesive interactions and axonal tension in grasshopper embryos. J Neurosci 9:2678-2686.

Condic ML, Bentley D (1989b) Pioneer growth cone adhesion in vivo to boundary cells and neurons after enzymatic removal of basal lamina in grasshopper embryos. J Neurosci 9:2687-2696.

Condic ML, Letourneau PC (1997) Ligand-induced changes in integrin expression regulate neuronal adhesion and neurite outgrowth. Nature 389:852-856.

Cooper JA (1987) Effects of cytochalasin and phalloidin on actin. J Cell Biol 105:1473-1478.

Davenport RW, Dou P, Mills LR, Kater SB (1996) Distinct calcium signaling within neuronal growth cones and filopodia. J Neurobiol 31:1-15.

Desai CJ, Gindhart JR, Goldstein LSB, Zin K (1996) Receptor tyrosine phosphatases are required for motor axon guidance in the Drosophila embryo. Cell 84:599-604.

Dubreuil RR, MacVicar G, Dissanayake S, Liu C, Homer D, Hortsch M (1996) Neuroglian-mediated cell adhesion induces assembly of the membrane skeleton at cell contact sites. J Cell Biol 133:647-655.

Forscher P, Smith SJ (1988) Actions of cytochalasins on the organization of actin filaments and microtubules in a neuronal growth cone. J Cell Biol 107:1505-1516.

Gallo G, Lefcort FB, Letourneau PC (1997) The trkA receptor mediates growth cone turning toward a localized source of nerve growth factor. J Neurosci 17:5445-5454.

Gomez TM, Letourneau PC (1994) Filopodia initiate choices made by sensory neuron growth cones at laminin/fibronectin borders in vitro. J Neurosci 14:5959-5972.

Gomez TM, Snow DM, Letourneau PC (1995) Characterization of spontaneous calcium transients in nerve growth cones and their effect on growth cone migration. Neuron 14:1233-1246.

Gundersen RW (1987) Response of sensory neurites and growth cones to patterned substrata of laminin and fibronectin in vitro. Dev Biol 121:423-431.

Gundersen RW (1988) Interference reflection microscopic study of dorsal root growth cones on different substrates: assessment of growth cone-substrate contacts. J Neurosci Res 21:298-306.

Hall A (1998) Rho GTPases and the actin cytoskeleton. Science 279:509-514.

He Q, Dent EW, Meiri KF (1997) Modulation of actin filament behaviour by GAP-43 (neuromodulin) is dependent on the phosphorylation status of serine 41, the protein kinase C site. J Neurosci 17:3515-3524.

Heidemann SR, Lamoureux P, Buxbaum RE (1990) Growth cone behaviour and production of traction force. J Cell Biol 111:1949-1957.

Hortsch M, Wang Y-ME, Marikar Y, Bieber AJ (1995) The cytoplasmic domain of the Drosophila cell adhesion molecule neuroglian is not essential for its homophilic adhesive properties. J Biol Chem 270:18809-18817.

Jan LY, Jan YN (1982) Antibodies to horseradish peroxidase as specific neural markers in Drosophila and grasshopper embryos. Proc Natl Acad Sci USA 79:2700-2704. 
Kater SB, Rehder V (1995) The sensory-motor role of growth cone filopodia. Curr Opin Neurobiol 5:68-74.

Kim Y-t, Wu C-f (1996) Reduced growth cone motility in cultured neurons from Drosophila memory mutants with a defective cAMP cascade. J Neurosci 16:5593-5602.

Kolodkin Al, Matthes DJ, O'Connor TP, Pate NH, Admon A, Bentley D, Goodman CS (1992) Fasciclin IV: sequence, expression and function during growth cone guidance in the grasshopper embryo. Neuron 9:831-845.

Kreft B, Berndoff D, Bottinger A, Finnemann S, Wedlich D, Hortsch M, Tauber R, Gebner R (1997) LI-cadherin-mediated cell-cell adhesion does not require cytoplasmic interactions. J Cell Biol 136:1109-1121.

Krueger NX, Van Vactor D, Wan HI, Gelbart WM, Goodman CS, Saito H (1996) The transmembrane tyrosine phosphatase DLAR controls motor axon guidance in Drosophila. Cell 84:611-622.

Kuhn TB, Schmidt MF, Kater SB (1995) Laminin and fibronectin guideposts signal sustained but opposite effects to passing growth cones. Neuron 14:275-285.

Kuhn TB, Wilcox CL, Bamburg JL (1997) RAC1, a small GTPase, alters F-actin organization in motor neuron growth cones regulating both extension and differentiation of neurites. Soc Neurosci Abstr 23:603.

Kuhn TB, Williams CV, Dou P, Kater SB (1998) Laminin directs growth cone navigation via two temporally and functionally distinct calcium signals. J Neurosci 18:184-194.

Lamoureux P, Zheng J, Buxbaum RE, Heidemann SR (1992) A cytomechanical investigation of neurite growth on different culture surfaces. J Cell Biol 118:655-661.

Lauffenburger DA, Horwitz AF (1996) Cell migration: a physically integrated molecular process. Cell 84:359-369.

Lemmon V, Burden S, Payne HR, Elmslie GJ, Hlavin ML (1992) Neurite growth on different substrates: permissive versus instructive influences and the role of adhesive strength. J Neurosci 12:818-826.

Letourneau PC (1975) Cell-to-substratum adhesion and guidance of axonal elongation. Dev Biol 44:92-101.

Letourneau PC, Shattuck TA, Ressler AH (1987) "Pull" and "push" in neurite elongation: observations on the effects of different concentrations of cytochalasin B and taxol. Cell Motil Cytoskeleton 8:193-209.

Lewis AK, Bridgman PC (1992) Nerve growth cone lamellipodia contain two populations of actin filaments that differ in organization and polarity. J Cell Biol 119:1219-1243.

Lin C-H, Forscher P (1995) Growth cone advance is inversely proportional to retrograde F-actin flow. Neuron 14:763-771.

Lin C-H, Thompson CA, Forscher P (1994) Cytoskeletal reorganization underlying growth cone motility. Curr Opin Neurobiol 4:640-647.

Luo L, Jan LY, Jan Y-n (1997) Rho family small GTP-binding proteins in growth cone signalling. Curr Biol 7:81-86.

MacLean-Fletcher S, Pollard TD (1980) Mechanism of action of cytochalasin B on actin. Cell 20:329-341.

Marsh L, Letourneau PC (1984) Growth of neurites without filopodial or lamellipodial activity in the presence of cytochalasin B. J Cell Biol 99:2041-2047.

Mitchison TJ, Cramer LP (1996) Actin-based cell motility and cell locomotion. Cell 84:371-379.

Myers PZ, Bastiani MJ (1993) Growth cone dynamics during the migration of an identified commissural growth cone. J Neurosci 13:127-143.

O'Connor TP, Bentley D (1993) Accumulation of actin in subsets of pioneer growth cone filopodia in response to neural and epithelial guidance cues in vivo. J Cell Biol 123:935-948.

O'Connor TP, Duerr JS, Bentley D (1990) Pioneer growth cone steering decisions mediated by single filopodial contacts in vivo. J Neurosci 10:3935-3946.

Regen CM, Horwitz AF (1992) Dynamics of b1 integrin-mediated adhesive contacts in motile fibroblasts. J Cell Biol 117:1321-1330.

Sabry JH, O'Connor TP, Evans L, Toroian-Raymond A, Kirschner M, Bentley D (1991) Microtubule behaviour during guidance of pioneer neuron growth cones in situ. J Cell Biol 115:381-395.

Saffell JL, Williams EJ, Mason IJ, Walsh FS, Doherty P (1997) Expression of a dominant negative FGF receptor inhibits axonal growth and FGF receptor phosphorylation stimulated by CAMs. Neuron 18:231-242.

Sampath P, Pollard TD (1991) Effects of cytochalasins, phalloidin, and $\mathrm{pH}$ on the elongation of actin filaments. Biochemistry 30:1972-1980.

Sanchez D, Ganfornina MD, Bastiani MJ (1995) Contributions of an orthopteran to the understanding of neuronal pathfinding. Immunol Cell Biol 73:565-574.

Schliwa M (1982) Action of cytochalasin D on cytoskeletal networks. J Cell Biol 92:79-91.

Schmidt CE, Horwitz AF, Lauffenburger DA, Sheetz MP (1993) Integrin-cytoskeletal interactions in migrating fibroblasts are dynamic, asymmetric, and regulated. J Cell Biol 123:977-991.

Shapiro L, Fannon AM, Kwong PD, Thompson A, Lehmann MS, Grubel G, Legrand JF, Als JJ, Colman DR, Hendrickson WA (1995) Structural basis of cell-cell adhesion by cadherins. Nature 374:327-337.

Song H-j, Ming G-1, Poo M-m (1997) cAMP-induced switching in turning direction of nerve growth cones. Nature 388:275-279.

Tsai A, Wong S, Isbister C, Kolodkin AL, O'Connor TP (1997) Characterization of grasshopper semaphorin II. Soc Neurosci Abstr 23:613.

Williams EJ, Doherty P, Turner G, Reid RA, Henperly JJ, Walsh FS (1992) Calcium influx into neurons can solely account for cell contactdependent neurite outgrowth stimulated by transfected L1. J Cell Biol 119:883-892.

Wong JTW, Yu WTC, O'Connor TP (1997) Transmembrane grasshopper semaphorin I promotes axon outgrowth in vivo. Development 124:3597-3607.

Zheng JQ, Wan J-j, Poo M-m (1996) Essential role of filopodia in chemotropic turning of nerve growth cone induced by a glutamate gradient. J Neurosci 16:1140-1149. 